\title{
Nachwuchsforschung im Blickpunkt - Metallurgie und Werkstofftechnik
}

Online publiziert am 30. August 2012

Sehr geehrte Leserinnen und Leser,

alle Originalbeiträge dieses BHM Heftes berichten über ausgewählte Forschungsarbeiten, die im Rahmen von Masterarbeiten und Dissertationen auf dem Gebiet der Metallurgie und Werkstofftechnik durchgeführt wurden. Die Arbeiten der jungen Forscherinnen und Forscher geben Ihnen einen Einblick über die beachtliche Themenbreite und das hohe wissenschaftlich-technische Niveau auf diesem Fachgebiet in Österreich. Die meisten dieser Arbeiten wurden im Rahmen des Forums für Metallurgie und Werkstofftechnik im Mai dieses Jahres in einer Vortragsreihe präsentiert. Die Resonanz des anwesenden Fachpublikums war sehr positiv. Wir Herausgeber möchten in Zukunft die BHM verstärkt als eine Plattform für junge Forscherinnen und Forscher anbieten, wo diese erste Erfahrungen mit der Veröffentlichung ihrer wissenschaftlichen Arbeiten sammeln können. Mit diesem Heft soll ein Startpunkt dafür gesetzte werden und wir hoffen, dass dies auch Ihrem Interesse entspricht.

Das 157-jährige Bestehen der BHM begründet sich maßgeblich durch die Qualität des Inhaltes aber auch dadurch, dass diese an neue Anforderungen und Rahmenbedingungen immer wieder angepasst wurde. Um uns ein Bild über die Lesermeinung zu den Metallurgie- und Werkstoffheften der BHM machen zu können, wurde Ende Mai an die ASMET Mitglieder ein elektronischer Fragebogen verschickt. Die überdurchschnittlich hohe Rücklaufquote von über $31 \%$ zeigt uns, wie wichtig Ihnen als Leserinnen und Leser diese Fachzeitschrift ist. Mehr als $72 \%$ der Antworten bewerteten die Hefte mit sehr gut oder gut, und es gab keine Beurteilung mit nicht zufriedenstellend. Des Weiteren haben wir Ihre Lesegewohnheit der BHM sowie Ihre Meinung zur Häufigkeit und Art der Publikation abgefragt. Das Ergebnis dieser Umfrage ist uns eine große Hilfe für die Entscheidungen, wie die BHM in Zukunft gestaltet werden soll. Wir bedanken uns bei allen, die sich an der Umfrage beteiligt haben. Alle ASMET Mitglieder können das Ergebnis dieser Umfrage auf der Homepage der ASMET nachlesen. (http://www.asmet.at/index.php/ de/news/389-auswertung-der-qbhm-befragungq-vom-21mai-2012)

\section{Mit herzlichem Glückauf!}

Helmut Clemens · Bruno Hribernik · Birgit Knoll ·

Johannes Schenk

Montanuniversität Leoben

Leoben, Österreich

E-Mail: Birgit.Knoll@unileoben.ac.at 